\title{
Contrast-Enhanced Magnetic Resonance Microangiography Reveals Remodeling of the Cerebral Microvasculature in Transgenic ArcA $\beta$ Mice
}

\author{
Jan Klohs, ${ }^{1 \star}$ Christof Baltes, ${ }^{1 \star}$ Felicitas Princz-Kranz, ${ }^{1}$ David Ratering, ${ }^{1}$ Roger M. Nitsch, ${ }^{2}$ Irene Knuesel, ${ }^{3}$ \\ and Markus Rudin 1,3 \\ ${ }^{1}$ Institute for Biomedical Engineering, Swiss Federal Institute of Technology and University of Zurich, CH-8093 Zurich, Switzerland, and ${ }^{2}$ Division of \\ Psychiatry Research, University of Zurich, CH-8008 Zurich, Switzerland, and ${ }^{3}$ Institute of Pharmacology and Toxicology, University of Zurich, CH-8057 \\ Zurich, Switzerland
}

Amyloid- $\beta(\mathrm{A} \beta)$ deposition in the cerebral vasculature is accompanied by remodeling which has a profound influence on vascular integrity and function. In the current study we have quantitatively assessed the age-dependent changes of the cortical vasculature in the $\operatorname{arcA} \beta$ model of cerebral amyloidosis. To estimate the density of the cortical microvasculature in vivo, we used contrast-enhanced magnetic resonance microangiography (CE- $\mu \mathrm{MRA})$. Three-dimensional gradient echo datasets with $60 \mu \mathrm{m}$ isotropic resolution were acquired in 4- and 24-month-old arcA $\beta$ mice and compared with wild-type (wt) control mice of the same age before and after administration of superparamagnetic iron oxide nanoparticles. After segmentation of the cortical vasculature from difference images, an automated algorithm was applied for assessing the number and size distribution of intracortical vessels. With CE- $\mu$ MRA, cerebral arteries and veins with a diameter of less than the nominal pixel resolution $(60 \mu \mathrm{m})$ can be visualized. A significant age-dependent reduction in the number of functional intracortical microvessels (radii of 20-80 $\mu \mathrm{m}$ ) has been observed in 24-month-old arcA $\beta$ mice compared with age-matched wt mice, whereas there was no difference between transgenic and wt mice of 4 months of age. Immunohistochemistry demonstrated strong fibrinogen and $\mathrm{A} \beta$ deposition in small- and medium-sized vessels, but not in large cerebral arteries, of 24 -monthold $\operatorname{arcA} \beta$ mice. The reduced density of transcortical vessels may thus be attributed to impaired perfusion and vascular occlusion caused by deposition of $\mathrm{A} \beta$ and fibrin. The study demonstrated that remodeling of the cerebrovasculature can be monitored noninvasively with CE- $\mu$ MRA in mice.

\section{Introduction}

Cerebral amyloid angiopathy (CAA) results from the failure to eliminate amyloid- $\beta(\mathrm{A} \beta)$ and other amyloid peptides from the cerebral vasculature in aged people and patients with Alzheimer's disease (AD; Glenner et al., 1981; Weller et al., 2008, 2009). A $\beta$ accumulates predominantly in cerebral microvessels such as capillaries and arterioles (Fischer at al., 1990; Buee et al., 1994; Miao et al., 2005; Kouznetsova et al., 2006) where it leads to an impeded cerebral blood supply, reduced vascular reactivity, and increased susceptibility to cerebral ischemia (Gilbert and Vinters, 1983;

\footnotetext{
Received Nov. 8, 2011; accepted Nov. 28, 2011

J.K., C.B., R.M.N., and M.R. designed research; J.K., C.B., F.P.-K., and I.K. performed research; C.B., D.R., and R.M.N. contributed unpublished reagents/analytic tools; J.K., C.B., F.P.-K., D.R., I.K., and M.R. analyzed data; J.K. and M.R. wrote the paper.

This work was funded by the Swiss National Science Foundation (Grants 3100A0-112835 and 310030-126029 to M.R. and 310030-132629 to I.K.). We gratefully acknowledge Daniel Schuppli (Division of Psychiatry Research, University of Zurich) for providing the arc $A \beta$ mice.

*J.K. and C.B. contributed equally to this work.

The authors declare no competing financial interests.

Correspondence should be addressed to Dr. Markus Rudin, Institute for Biomedical Engineering, University of Zurich and ETH, AIC-ETH HCI D426, Wolfgang-Pauli-Strasse 10, CH-8093 Zürich, Switzerland. E-mail: rudin@biomed.ee.ethz.ch.

DOI:10.1523/JNEUROSCI.5626-11.2012

Copyright $\odot 2012$ the authors $\quad 0270-6474 / 12 / 311705-09 \$ 15.00 / 0$
}

Cadavid et al., 2000; Haglund et al., 2006; Ryu and McLarnon, 2009). These changes in hemodynamic conditions result in longterm structural changes known as vascular remodeling. Blood vessels become tortuous, the composition of the basement membrane is altered (de la Torre, 1997; Moody et al., 1997), and the overall vascular density decreases (Lee et al., 2005; Bouras et al., 2006). Furthermore, changes in the proteolytic microenvironment lead to impairment of blood-brain-barrier (BBB) integrity and the occurrence of cerebral microbleeds (CMBs) and intracerebral hemorrhage (Gilbert and Vinters, 1983; Franceschi et al., 1995; Ryu and McLarnon, 2009). There is increasing evidence that vascular remodeling might not only reflect an adaptive process, but also constitutes an important mechanism in the pathophysiology of AD and CAA (Weller et al., 2009; Marchesi, 2011).

Cerebrovascular $\mathrm{A} \beta$ deposition can be mimicked in mouse models, which, therefore, have become central for studying the pathophysiology of CAA and evaluating novel therapeutic approaches (Calhoun et al., 1999; Herzig et al., 2004; Miao et al., 2005; Kouznetsova et al., 2006). Recently, the $\operatorname{arcA} \beta$ mouse line has been generated, which shows age-dependent cerebrovascular dysfunction as reflected by BBB impairment, neurovascular uncoupling (Merlini et al., 2011), decreased vascular reactivity (Princz-Kranz et al., 2010), and the occurrence of cerebral mi- 

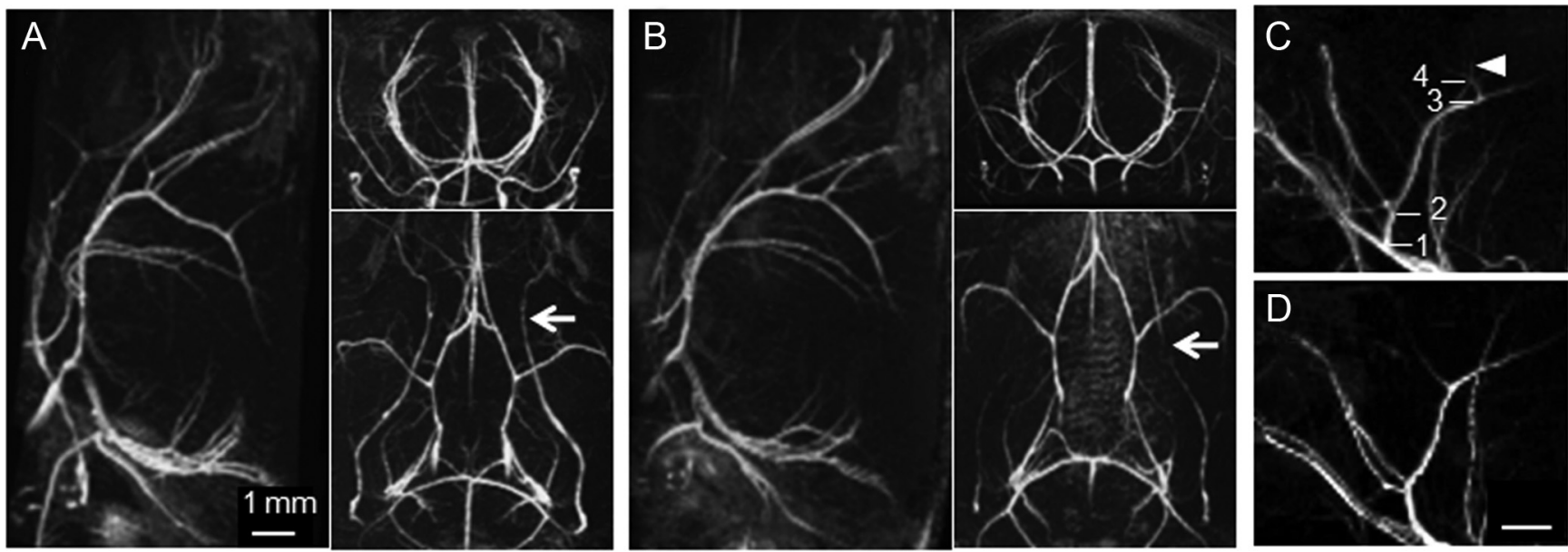

Figure 1. High-resolution 3D TOF-MRA of the intracranial and extracranial vasculature of a 24-month-old wt control mouse $(\boldsymbol{A})$ and an age-matched arcA $\beta$ mouse $(\boldsymbol{B})$. The representative maximal intensity projections show the angiograms in sagittal, axial, and horizontal views. While the intracranial vasculature of arc $A \beta$ and wt mice shows no flow disturbances, flow voids are seen in extracranial vessels (white arrows). Sections of MIPs of the anterior cerebral artery of a 4-month-old $(\boldsymbol{C})$ and a 24-month-old $(\boldsymbol{D})$ wt control mouse. Discernable bifurcations are numbered in hierarchical order. More distal branches are visible in the angiogram of the 4-month-old mouse ( $\boldsymbol{C}$, white arrowhead). Scale bar, $1 \mathrm{~mm}$.

crobleeds (Klohs et al., 2011; Merlini et al., 2011). Based on these findings, we hypothesized that the arcA $\beta$ mice would show CAArelated remodeling of the cerebral microvasculature. We used high-resolution contrast-enhanced magnetic resonance angiography (CE- $\mu \mathrm{MRA}$ ) in 4 - and 24 -month-old $\operatorname{arcA} \beta$ mice and agematched wild-type (wt) control mice to assess age-dependent changes in the cerebral vasculature. Aged arcA $\beta$ mice exhibited a significant reduction in density of functional intracortical microvessels with radii in the range of $20-80 \mu \mathrm{m}$, while large cerebral vessels, as detected with conventional time-of-flight MRA (TOF-MRA), were not affected. This in vivo observation is in line with results of the immunohistochemistry revealing that $\mathrm{A} \beta$ and fibrinogen are predominantly deposited in small- and medium-sized vessels, but spare larger cerebral vessels. Hence, the reduced density of intracortical vessels observed in advanced disease may be attributed to obstruction of perfusion as a result of abnormal fibrinolysis. In murine models of cerebral amyloidosis, the remodeling of these vascular structures can be monitored noninvasively with CE- $\mu$ MRA.

\section{Materials and Methods}

Animals. All experiments were performed in accordance with the Swiss Federal Act on Animal Protection. We used $\operatorname{arc} A \beta$ transgenic mice of either sex, which express the human APP 695 containing both the Swedish and the Arctic mutation under the control of the prion protein promoter as described previously (Knobloch et al., 2007). Age- and gendermatched wt littermates served as controls. Mice at the age of 4 months $(\operatorname{arcA} \beta, n=4$; wt, $n=4)$ and 24 months $(\operatorname{arcA} \beta, n=6$; wt, $n=5)$ were used for the study. Animals were kept at standard housing conditions (temperature, $20-24^{\circ} \mathrm{C}$; relative humidity, minimum $40 \%$; light/dark cycle, $12 \mathrm{~h}$ ) providing water and food ad libitum.

Magnetic resonance imaging. Experiments were performed on a Bruker BioSpec 94/30 (Bruker BioSpin) small animal MR system operating at $400 \mathrm{MHz}$ with a gradient system, capable of a maximum gradient strength of $400 \mathrm{mT} / \mathrm{m}$ with an $80 \mu$ s rise time. A cryogenic quadrature RF surface coil (Bruker BioSpin) was used for RF signal transmission and reception (Baltes et al., 2009). Anesthesia was induced with $3 \%$ isoflurane (Abbott) in a 4:1 air/oxygen mixture and maintained with $1.2 \%$ isoflurane. Mice were endotracheally intubated and mechanically ventilated with 90 breaths/min, applying a respiration cycle of $25 \%$ inhalation and 75\% exhalation (MRI-1 Volume Ventilator, CWI). Temperature was kept at $36.0 \pm 0.5^{\circ} \mathrm{C}$ using a warm-water circuit integrated into the animal support (Bruker BioSpin). Body temperature was monitored with a rectal temperature probe (MLT415, ADInstruments). The tail veins of the mice were cannulated for administration of contrast agents. Tri-pilot scans were used for accurate positioning of the animal head inside the magnet. For high-resolution structural imaging, we used a 2D FLASH sequence with the parameter $\mathrm{T}_{\mathrm{E}} / \mathrm{T}_{\mathrm{R}}=8 / 345 \mathrm{~ms}$; RF pulse angle, $\alpha=30^{\circ}$; bandwidth $=50 \mathrm{kHz}$; number of averages (NA) $=1$. Fifteen 0.5 -mm-thick slices with an interslice distance of $0.6 \mathrm{~mm}$ were acquired with a field of view (FOV) of $20 \times 20 \mathrm{~mm}^{2}$ and matrix dimension of $384 \times 384$, resulting in a spatial resolution of $52 \times 52 \mu \mathrm{m}^{2}$. For TOFMRA a 3D FLASH sequence with the parameters $T_{E} / T_{R}=2.5 / 15 \mathrm{~ms}$, $\alpha=30^{\circ}$, bandwidth $=98 \mathrm{kHz}, \mathrm{NA}=2$ was used. A horizontal slab of $19.8 \times 15.9 \times 8 \mathrm{~mm}^{3}$ was recorded using a matrix dimension of $248 \times$ $199 \times 100$, resulting in isotropic voxel dimensions of $80 \mu \mathrm{m}^{3}$. The acquisition time was $7 \mathrm{~min}$ and $27 \mathrm{~s}$. For CE- $\mu \mathrm{MRA}$, precontrast and postcontrast images were acquired using a 3D FLASH sequence with $\mathrm{T}_{\mathrm{E}} / \mathrm{T}_{\mathrm{R}}=2.9 / 150 \mathrm{~ms}, \alpha=20^{\circ}$, bandwidth $=82 \mathrm{kHz}, \mathrm{NA}=2$. A slab of $15 \times 12 \times 2.2 \mathrm{~mm}^{3}$ was recorded with matrix dimensions of $248 \times$ $199 \times 36$ with a spatial resolution of $60 \times 60 \times 61 \mu \mathrm{m}^{3}$. Animals were injected intravenously with $50 \mu \mathrm{l}$ of an iron-oxide contrast agent (corresponding to $0.52 \mathrm{mg}$ of Fe; Endorem, Guerbet) as a bolus into the tail vein. The postcontrast image acquisition was started $1 \mathrm{~min}$ after the injection of the contrast agent. The acquisition time was $36 \mathrm{~min}$ for each scan.

Determination of contrast-to-noise ratios. Regions of interest (ROIs) were drawn in vessels and adjacent tissue of precontrast images. A noise ROI was placed outside the head of the animal. Signal-to-noise (SNR) ratios were calculated by dividing the signal intensity of the vessel ROI by the SD of the noise ROI. SNR values were calculated for four regions separately and then averaged.

Reconstruction of angiograms. For TOF-MRA datasets, angiograms were generated by maximum intensity projections (MIPs) using Paravision 5.0 (Bruker BioSpin MRI). All angiograms were evaluated by investigators blinded to the genotype of the mouse. MIPs of TOF-MRA were inspected from various projection angles. Flow voids were detected when the signal intensities in an identified blood vessel reached background level. Distality was counted by assessing the number of visible branches of the anterior cerebral artery (Fig. 1C,D). Distality count, i.e., the number of hierarchical levels of vascular branches detected, was only estimated for MIPs of TOF-MRA data, because for CE- $\mu$ MRA the whole vessel tree was not covered in the acquisition.

For CE- $\mu \mathrm{MRA}$, difference images were first obtained by subtraction of the postcontrast image from the precontrast image before MIP generation using home-written software based on IDL (Interactive Data Language, version 6.4; ITT Visual Information Solutions).

Assessment of vessel number and vessel radius. For segmentation of the cerebral cortex, ROIs were drawn for each animal on each $61-\mu \mathrm{m}$-thick 

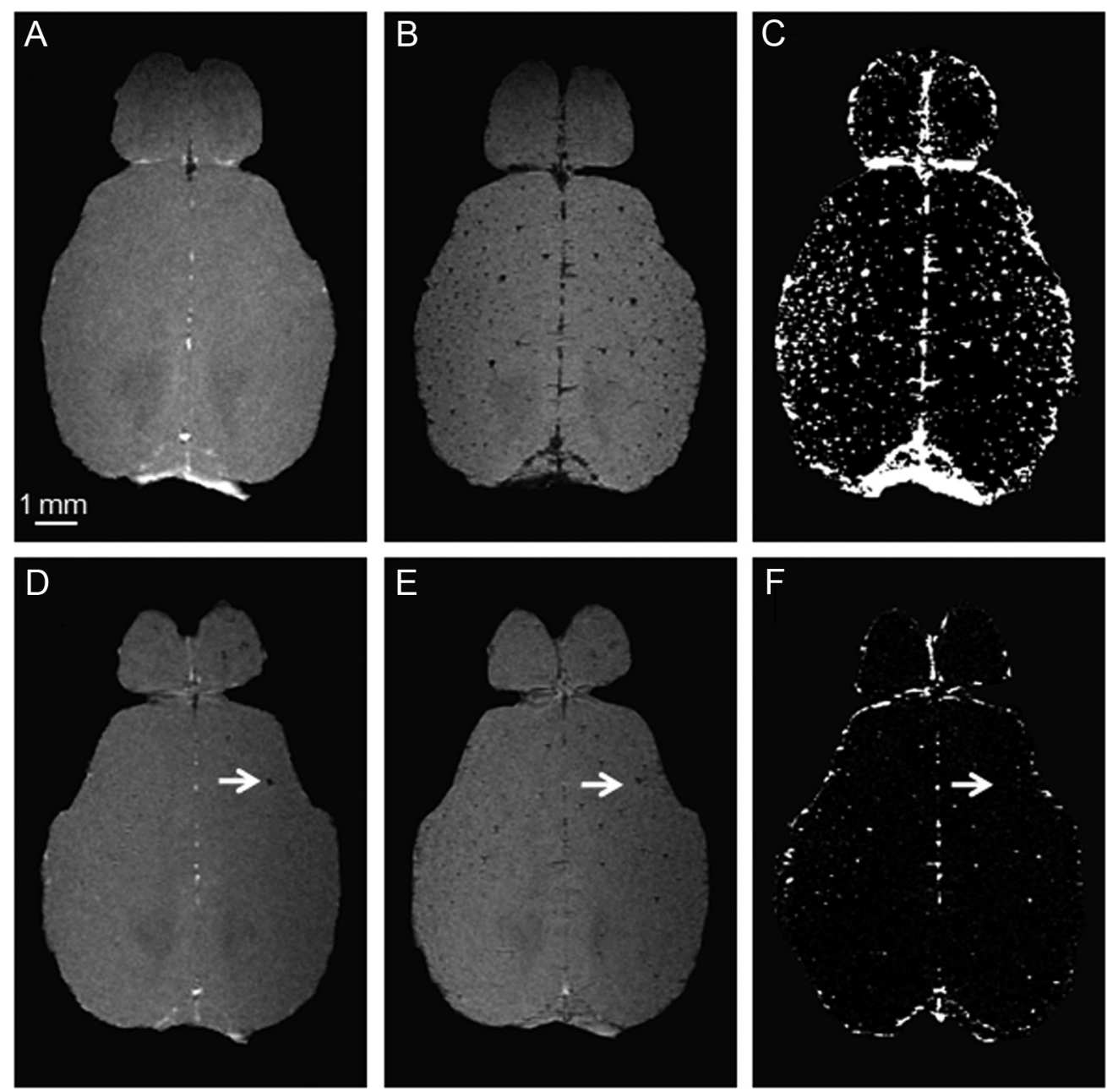

Figure 2. Precontrast $(\boldsymbol{A}, \boldsymbol{D})$ and postcontrast $(\boldsymbol{B}, \boldsymbol{E})$ images were acquired before and after administration of a superparamagnetic iron oxide contrast agent. Grayscale windowing was kept identical for both images. Difference images $(\boldsymbol{C}, \boldsymbol{F})$ are obtained by subtraction of the postcontrast image from the precontrast image. Depicted are representative horizontal images of the cortex and olfactory bulb of a 24-month-old wt mouse $(\boldsymbol{A}-\boldsymbol{C})$ and an age-matched arcA $\beta$ mouse $(\boldsymbol{D}-\boldsymbol{F})$. After administration of the contrast agent, hypointensities representing intact blood vessels become visible which are not discernable on the precontrast image. The arrows point to focal hypointense areas that are present before administration of the contrast agent. Scale bar: $1 \mathrm{~mm}$.

slice of the 3D dataset. The ROIs were chosen to encompass both hemispheres of the neocortex over a depth of $430 \mu \mathrm{m}$. To exclude meningeal vasculature, superficial brain slices to a depth of $350 \mu \mathrm{m}$ below the brain surface were not considered in the ROI analysis. A noise ROI was placed outside the brain. The selected ROIs were combined to define a volume of interest (VOI) required for the subsequent $3 \mathrm{D}$ vessel analysis. In this algorithm, vessel structures were identified according to the following two threshold criteria: A voxel was considered to belong to a vessel, if (1) the signal of the voxel was higher than the signal threshold $\left(S_{\text {Threshold }}\right)$ as defined in Equation 1, and (2) the number of connected voxels was equal to or higher than the connectivity threshold. $S_{\text {Threshold }}$ was defined according to

$$
S_{\text {Threshold }}=\bar{S}_{\text {ROI,Noise }}+t_{\text {Noise }} \cdot S D\left(S_{\text {ROI,Noise }}\right) \text {, }
$$

where $\bar{S}_{\text {ROI,Noise }}$ and $S D\left(S_{\text {ROI,Noise }}\right)$ define the average and the SD, respectively, of the signal in the noise ROI defined outside of the object of interest (in a corner of the field-of-view). The parameter $t_{\text {Noise }}$ could be chosen interactively. Unless indicated otherwise, we used a value of $t_{\text {Noise }}=8$.

For voxels fulfilling this threshold criterion, a 3D search algorithm was applied searching for voxels connected along edges or corners. Structures for which the number of connected voxels exceeded the connectivity threshold set to 3,6 , and 9 were considered as vessels.

The intensity of a vascular signal depends on the relative voxel volume taken by the vascular compartment. Correspondingly, evaluation of these partial volume effects allowed estimating the compartment size and thus an upper limit for the vessel dimensions. For this purpose we assumed a perpendicular orientation of the vessel with respect to the imaging slices. For transcortical vessels oriented radially with regard to the cortical surface, this approximation appears reasonable. Within each slice the voxel with the highest signal intensity $S_{k \text {, max }}$ in the difference images was assumed to comprise only the vascular compartment. Accordingly, the radius of the $i$-th cross-sectional vessel in the $k$-th imaging slice was calculated as

$$
r_{i k}=\sqrt{\frac{\sum_{v}\left(\frac{S_{i k v}}{S_{k, \max }}\right) \cdot A_{\text {Voxel }}}{\pi}}
$$

where $A_{\text {Voxel }}$ is the cross-sectional area of one voxel and the summation is running over all voxels $v$ in slice $k$ contributing to vessel $i$. The average vessel radius $r_{i}$ is then obtained by averaging the values $r_{i k}$ across all slices displaying the vessel.

Immunohistochemistry. All animals were deeply anesthetized by intraperitoneal injection of ketamine/xylazine $(100 / 20 \mathrm{mg} / \mathrm{kg}$ body weight) and perfused transcardially with PBS ( $\mathrm{pH} 7.4$ ), followed by $4 \%$ paraformaldehyde in PBS (Sigma-Aldrich Chemie). Brains were then embedded in paraffin and cut into $10-\mu \mathrm{m}$-thick horizontal sections. The sections were pretreated in $85^{\circ} \mathrm{C} \mathrm{Na}$-Citrate buffer $(0.1 \mathrm{M}, \mathrm{pH} 4.5$, in the microwave), followed by a 5 min submersion in $95 \%$ formic acid. Blocking of 

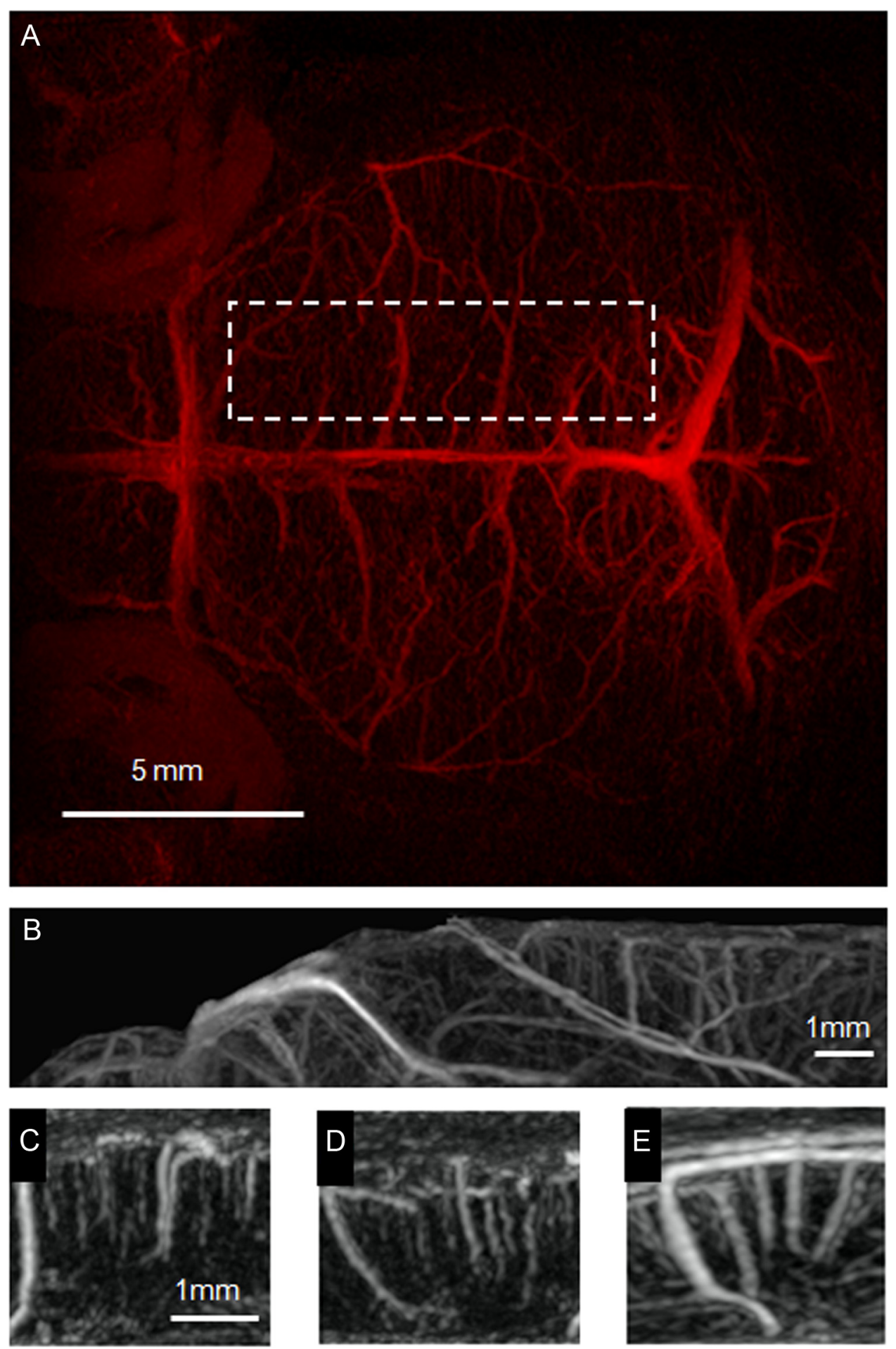

Figure 3. MIPs derived from a 3D stack of difference images viewed in horizontal $(\boldsymbol{A})$, sagittal $(\boldsymbol{B}-\boldsymbol{D})$, and axial $(\boldsymbol{E})$ orientation. The projections $\boldsymbol{A}$ and $\boldsymbol{B}$ were acquired over the whole acquisition volume, covering $2.2 \mathrm{~mm}$ of the upper part of the mouse brain. The horizontal view is dominated by projections of superficial veins like the transverse and superior sagittal sinus. Subcubes $\boldsymbol{C}-\boldsymbol{E}$ were taken from the axial MIP (location in the brain is indicated by the dashed line in $\boldsymbol{A}$ ). The cerebral vasculature is highly organized, running perpendicular to the cortical surface.

nonspecific binding was done in a mixture of $4 \%$ BSA, $5 \%$ normal goat serum, and $5 \%$ horse serum in PBS for $1 \mathrm{~h}$ at room temperature. The sections were then incubated overnight at $4^{\circ} \mathrm{C}$ in the primary antibody solution containing mouse antifibrinogen antibodies (Abcam, catalog \#52861, 1:1000) or rabbit anti-von Willebrand factor (vWF, Novocastra, catalog \#NCL-vWFp, 1:500) in PBS containing 2\% normal goat serum and $0.2 \%$ Triton X-100. After three washes in PBS, tissue sections were incubated for $30 \mathrm{~min}$ at room temperature in corresponding secondary antibodies coupled to Cy3 (diluted 1:500; Jackson ImmunoResearch).
Thioflavin S counterstaining involved $10 \mathrm{~min}$ incubation in filtered $1 \%$ aqueous thioflavin $\mathrm{S}$ (Sigma-Aldrich) solution at room temperature, followed by two washes for $5 \mathrm{~min}$ in $80 \% \mathrm{EtOH}, 5 \mathrm{~min}$ wash in $95 \% \mathrm{EtOH}$, and three 5 min washes in distilled water. Brain sections were then airdried in the dark and mounted with aqueous permanent mounting medium containing DAPI for nuclear counterstainings (DAKO).

Analysis of immunohistochemical stainings. Qualitative evaluation of the immunofluorescence labeling was done with a confocal microscope (LSM-710, Zeiss) using the $40 \times$ (NA1.3) objective. Double immunoflu- 
orescence staining was visualized using sequential acquisition of each channel. The pinhole aperture was set to 1.0 Airy unit for each channel. Stacks of consecutive optical sections $(6 ; 512 \times 512$ pixels, spaced $0.5 \mu \mathrm{m}$ in $z$ ) were acquired. For visual display, $Z$-sections of both channels were projected in the $z$-dimension (maximal intensity), and merged using the image analysis software Imaris (Bitplane). Cropping of images and adjustments of brightness and contrast were identical for each labeling and done using Adobe Photoshop (Adobe Systems).

Quantitative analyses of the vWF-immunoreactivity associated with the endothelial cells of the cerebral vasculature were done on three different horizontal brain sections obtained from 5 mice per age and genotype. Two digital images per brain hemisphere were acquired using a $40 \times$ (NA1.3) objective. The area covered by the immunoreactive signal was quantified using a threshold algorithm of ImageJ software. The binary images were segmented and the individual vessels outlined. The corresponding masks were analyzed with regard to the total area covered by the anti-vWF immunoreactivity. The different measures were averaged per animal and included in the statistical analysis.

For the quantitative evaluation of the fibrinogen accumulation and colocalization with thioflavin $\mathrm{S}$ associated with vascular plaques, three different brain sections per animal ( $n=5$ per age and genotype) were included. For each hemisphere, one digital tile-scan $(1.0 \times 0.76 \mathrm{~mm})$ was acquired using a $20 \times$ Apochromate objective (Zeiss, NA 0.75). First, red and green channels were individually segmented and then analyzed with regard to pixel overlap within outlined vessels using a colocalization plug-in of Image (NIH). The diameter of the vessels showing colocalized thioflavin S/fibrinogen immunoreactivity was recorded and the cumulative distribution and frequency calculated as a function of their size.

Statistical analysis. Data are presented as mean \pm SD. Student's $t$ test, 1-way ANOVA, or repeated-measures ANOVA followed by Tukey's test (SigmaStat 3.0, Systat Software) were used for statistical comparison, where appropriate. The statistical significance level was set to $p<0.05$.

\section{Results}

\section{Assessment of vessel status with angiograms}

To evaluate the effect of $A \beta$ deposition on major cerebral arteries, $3 \mathrm{D}$ TOF angiograms have been recorded in wt and $\operatorname{arcA} \beta$ mice at 4 months $(n=4$ each $)$ and 24 months $(n=6$ each) of age. In TOF-MRA the signal arises from fully magnetized blood that has entered the imaging volume and reached the respective vascular voxel in a defined time interval (flow encoding time), without the need of administering a contrast agent (Reese et al., 1999). MIPs displayed in different orientations allow clear identification of major intracranial arteries as illustrated for a 24-month-old wt and $\operatorname{arc} \mathrm{A} \beta$ mouse (Fig. 1 $A, B$ ). No apparent flow abnormalities could be detected in the vessels of wt control and $\operatorname{arcA} \beta$ mice at both ages. The distality count did not reveal any trait-specific difference at either age, yielding values of $4.2 \pm 0.4$ and $4.2 \pm 0.4$ for 4-month-old wt and $\operatorname{arcA} \beta$ mice and $3.5 \pm 0.4$ and $3.5 \pm 0.5$ for 24 -month-old wt and $\operatorname{arcA} \beta$ mice, respectively. The distality value tended to be slightly lower in aged animals, though the difference was not significant (Fig. 1C,D). Interestingly, flow voids were detected in extracranial vessels such as the pterygopalatine arteries of wt and $\operatorname{arcA} \beta$ mice at both ages (for 24 months, Fig. $1 A, B$, white arrows). Again, no difference was observed between wt and $\operatorname{arc} A \beta$ mice.

Visualization of vascular structures can be enhanced using intravascular contrast agents such as iron oxide nanoparticles, which, due to their large net positive magnetic moments, induce spin dephasing resulting in a net signal loss (Boxerman et al., 1995; Bolan et al., 2006). Figure 2 displays representative horizontal images of the brain of a 24-month-old wt control and an $\operatorname{arcA} \beta$ mouse. Following the administration of Endorem, transcortical blood vessels became visible as focal signal voids that could not be discerned on the precontrast image (Fig. $2 B, E$ ) and could be enhanced in difference images, as nonvascular brain
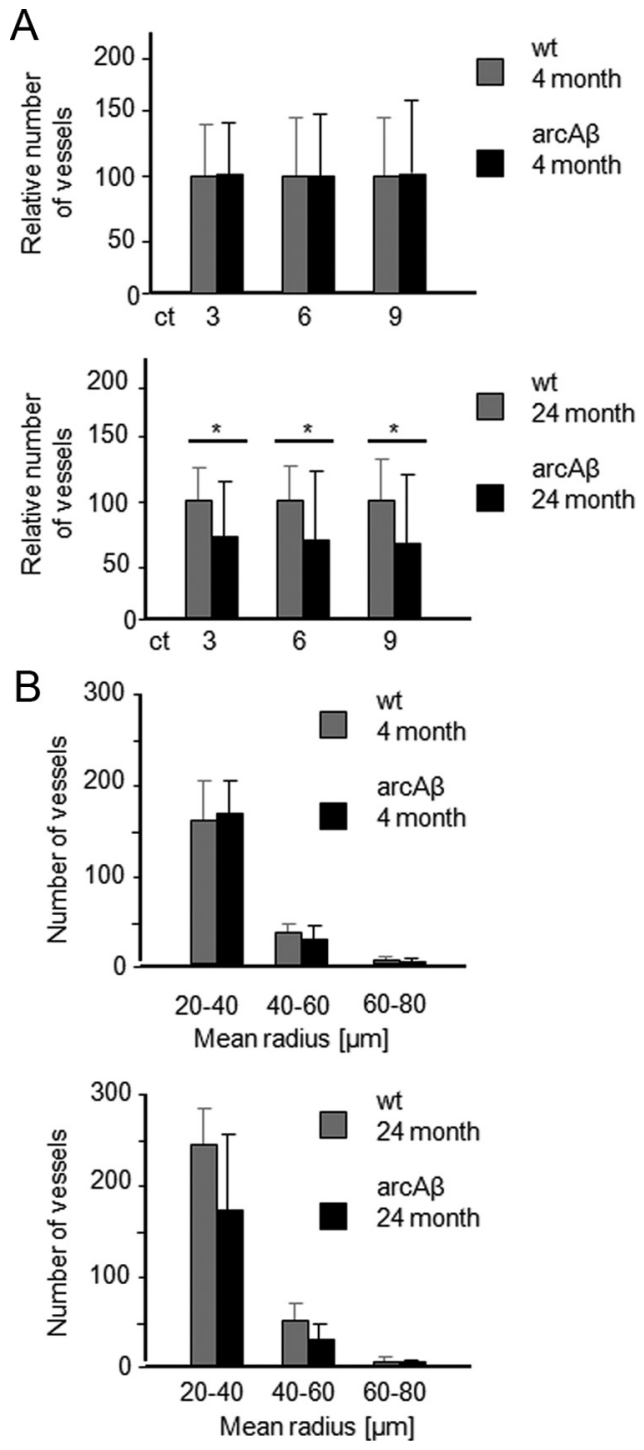

Figure 4. Semiautomated analysis of intracortical vessel density of wt and $\operatorname{arc} A \beta$ mice at 4 and 24 months of age. $\boldsymbol{A}$, The relative number of vessels was estimated for different connectivity thresholds corresponding to 3,6, and 9 pixels, corresponding to minimal length of a vessel segment of 183,366 , and $546 \mu \mathrm{m}$, respectively. $\boldsymbol{B}$, Number of vessels categorized according to their estimated vessel radius when the connectivity threshold was set to 3 . The algorithm counts the fraction of vessels above an intensity threshold for different values of vessel connectivity in a VOI covering the cortex. A significant decrease in the number of vessels was observed in 24-month-old arc $A \beta$ compared with wt controls, while no significant differences were seen between arcA $\beta$ mice and wt controls at 4 months of age (mean $\pm S D$; ${ }^{*} p<0.05$, repeatedmeasures ANOVA and Tukey's test). There was no significant difference between the number of vessels in each category among the four groups tested.

structures were canceling out (Fig. 2C,F). We noticed remarkable variations in the signal intensity of the vascular structures in the difference images. This is attributed to partial volume effects. This intensity information can, in turn, be used to estimate the size of the vascular compartment within a voxel, from which a maximum vessel diameter might be deduced (assuming that the vascular compartment is due to a single vessel crossing the voxel). Several large circular hypointensities (mean diameter of $242.5 \pm$ $108.1 \mu \mathrm{m}$ ) have been observed in the brains of 24 -month-old $\operatorname{arcA} \beta$ mice already before the administration of the contrast agent (Fig. $2 D$, white arrow). Such structures have not been observed in the brains of 4 -month-old $\operatorname{arcA} \beta$ mice and 4- and 24month-old wt control mice and represent most likely either large 

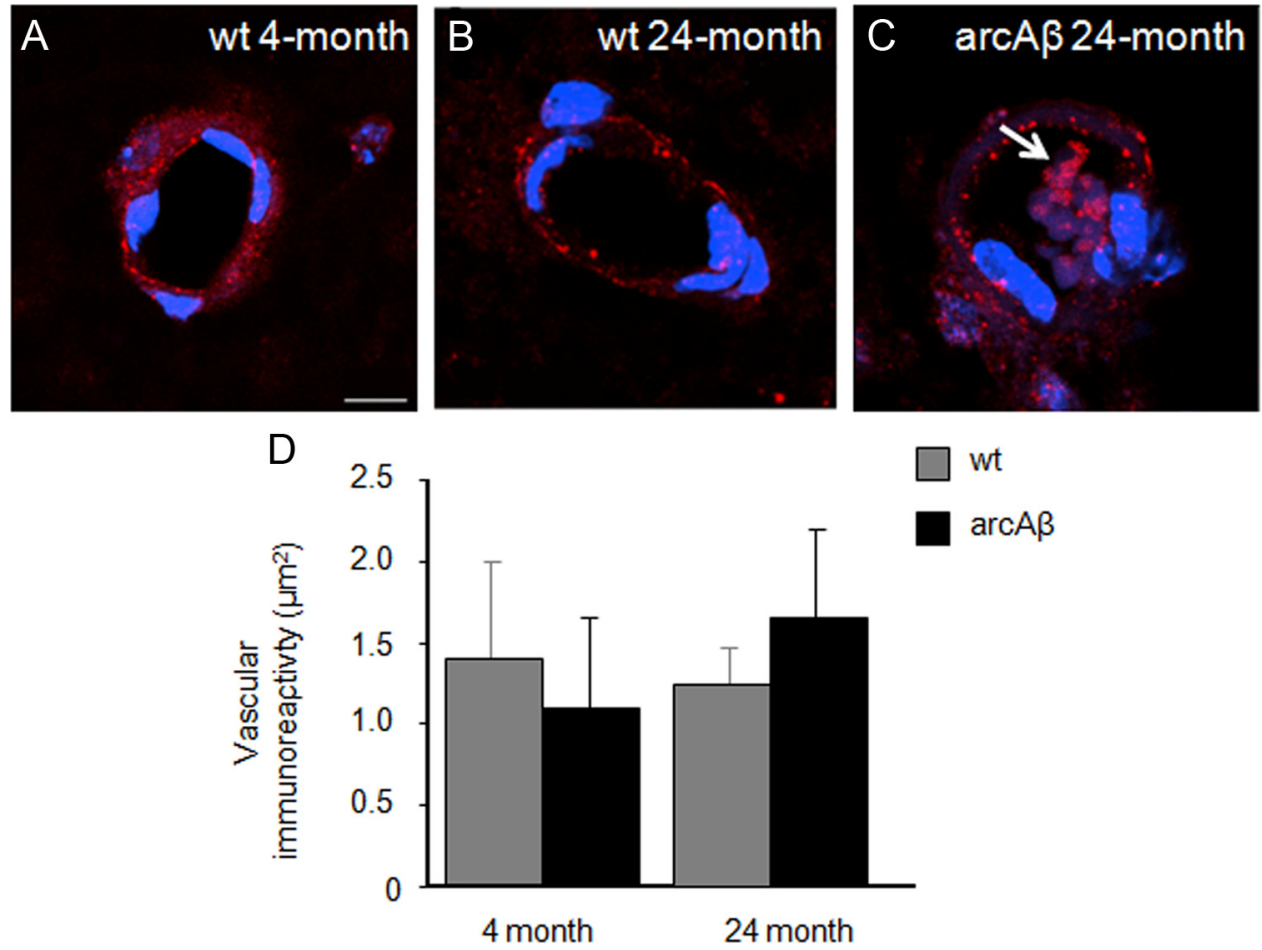

Figure 5. Assessment of the endothelial marker vWF (red) with immunohistochemistry. Nuclear staining was done with DAPI (blue). Representative confocal images of 4-month-old wt ( $A$ ), 24-month-old wt (B), and 24-month-old arc $\beta$ mice (C). The white arrow points to VWF immunoreactivity in thrombus-like structure in a microvessel of a 24 -month-old arc $A \beta$ mouse. There was no difference in vWF immunoreactivity between arcA $\beta$ and wt controls at 4 months of age and between arcA $\beta$ and wt controls at 24 months of age (D; mean \pm SD; $t$ test). Scale bar: $10 \mu \mathrm{m}$.

iron-containing A $\beta$ plaques (Benveniste et al., 1999) or cerebral microbleeds (Klohs et al., 2011). These structures disappear in the difference images, because they are not affected by the administration of the intravascular contrast agent (Fig. $2 F)$. In addition, in all animals few foci of reduced signal intensity have been detected in the precontrast images. These are attributed to venous structures for which the relatively high concentration of paramagnetic deoxyhemoglobin leads to decreased blood $\mathrm{T}_{2}{ }^{*}$ values already in the absence of an exogenous contrast agent (Ogawa et al., 1990; Reichenbach et al., 1997).

For illustration purposes, 3D stacks of difference images have been constructed for computing of MIPs (Fig. 3). Clearly, these MIPs provide significantly more detail about the vascular anatomy than those obtained from TOF angiograms, which essentially reveal only large arterial structures. While CE-MIPs are dominated by large vessels running at the brain surface, such as the transverse and superior sagittal sinus, many transcortical vessels become apparent (Fig. $3 A, B$ ). These can be highlighted by visualizing a cortical subcube of the full $3 \mathrm{D}$ dataset (Fig. $3 C-E$ ).

\section{CE- $\mu$ MRA reveals age-dependent decrease in transcortical vessels}

An algorithm was developed which assumes that blood vessels form a continuous tree structure. Structures that exceeded a userdefined intensity threshold in the 3D dataset derived from difference images were counted as vessels provided they fulfilled the connectivity criterion, which states that the vessel can be observed over multiple slices within the 3D dataset. The strength of this criterion could be adjusted. The number of vessels was estimated for different values of vessel connectivity i.e., 3,6 , and 9 pixels, corresponding to minimal length of a vessel segment of 183,366 , and $546 \mu \mathrm{m}$ respectively. Using this algorithm, individual datasets have been analyzed in a semiautomated fashion. The quantitative results on vessel counts are illustrated in Figure 4. We did not observe any difference in vessel count in 4-month-old wt and $\operatorname{arcA} \beta$ mice (Fig. $4 A$ ). However, there was a significant reduction in the number of transcortical vessels in 24-month-old transgenic compared with wt animals in a volume of $\sim 11 \mathrm{~mm}^{3}$ $\left({ }^{*} p<0.05\right)$. Vessels were categorized according to their vessel radius as estimated based on its signal intensity, which is governed by the fractional volume of the vascular compartment (Fig. $4 B$ ). There were no significant differences between the numbers of vessels in each category among the four groups, though the mean number of small $(20-40 \mu \mathrm{m})$ and medium sized $(40-60$ $\mu \mathrm{m})$ cortical vessels in the group of 24 -month-old $\operatorname{arcA} \beta$ mice tended to be smaller than in the respective group of wt animals.

\section{Abnormal fibrinolysis in small- and medium-sized thioflavin S-positive vessels}

Immunohistochemical assessment of vascular density, $A \beta$ and fibrinogen accumulation was performed on brain sections of 4 - and 24-month-old wt and arcA $\beta$ mice. Confocal analysis identified endothelial cells (Fig. 5A-C). Additional vWF immunoreactivity was revealed in thrombus-like structures in microvessels of 24-month-old arcA $\beta$ mice only (Fig. $5 C$, white arrow). Quantitative analysis of vWF immunoreactivity demonstrated no differences between the four groups investigated.

Thioflavin S-positive $\mathrm{A} \beta$ accumulation was apparent in cerebral vessels in sections of 24 -month-old $\operatorname{arcA} \beta$ mice (Fig. $5 C$ ), but was observed neither in sections of age-matched wt controls (Fig. $5 B$ ) nor in 4-month-old transgenic (Fig. 6A) and wt mice (data not shown). Investigation of different anatomical regions revealed a high abundance of thioflavin S-positive vessels in cortical areas of 24-month-old arcA $\beta$ mice, while vessels in the thalamus showed almost no $\mathrm{A} \beta$ deposition (results not shown). Autofluorescence of pigment granules were observed in 24-month-old wt 

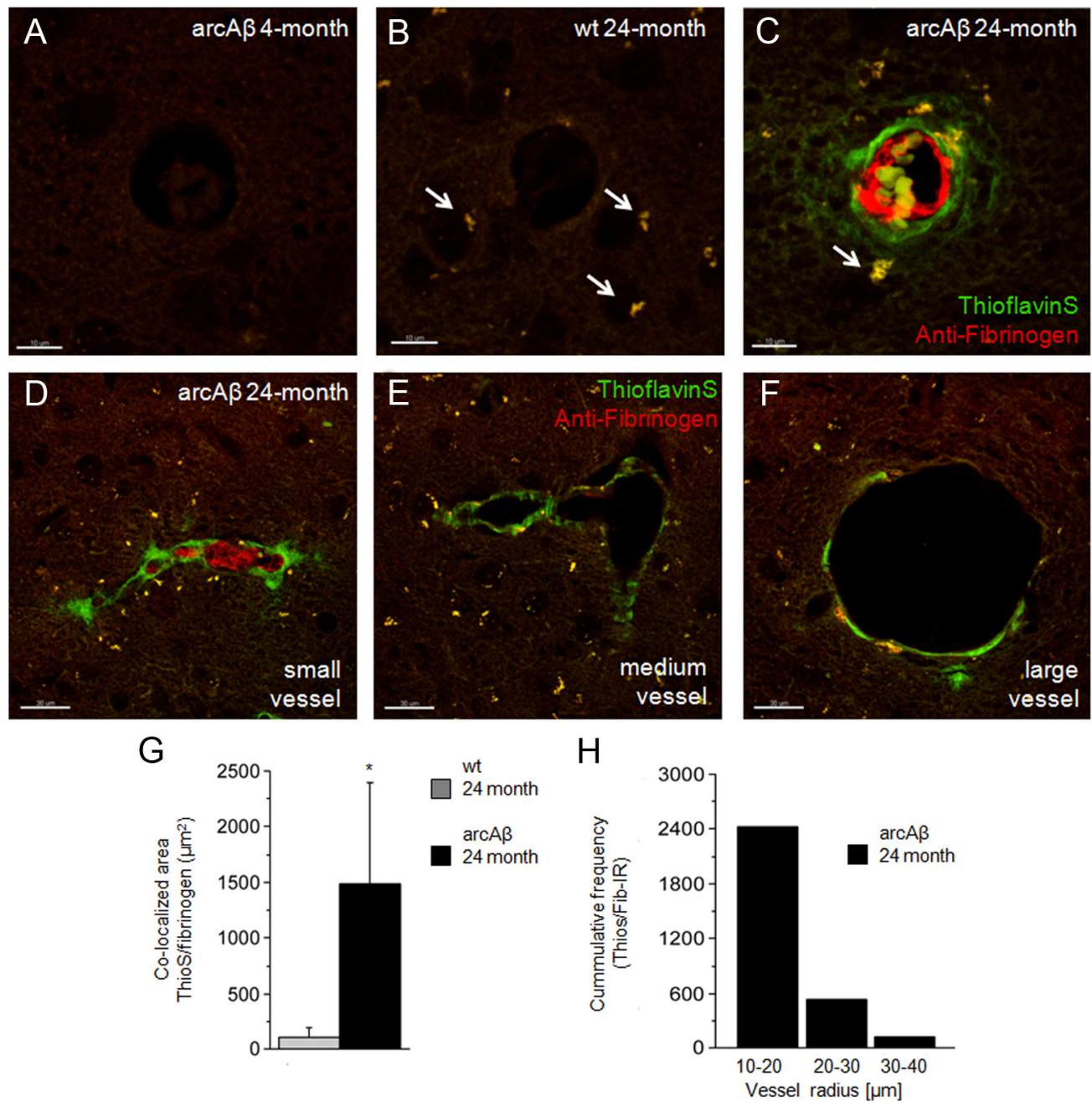

Figure 6. Assessment of $A \beta$ and fibrinogen deposition in wt and arc $A \beta$ mice. Depicted are representative confocal images of 4-month-old arc $A \beta(\boldsymbol{A})$, 24-month-old wt (B), and 24-month-old $\operatorname{arc} A \beta$ mouse brain sections $(\boldsymbol{C}-\boldsymbol{F})$ after thioflavin $S$ staining (green) and antifibrinogen immunohistochemistry (red). The white arrows point to autofluorescence caused by lipofuscin aggregates, which are typical findings in neurons of aged animals. Fibrinogen accumulation was seen only in thioflavin S-positive vessels in 24-month-old arcA $\beta$ mice. Fibrinogen accumulation colocalized strongly with $A \beta$ deposition ( $G$; mean $\pm S D ;{ }^{*} p<0.05$ Student's $t$ test). Deposition of $A \beta$ and fibrinogen were confined to small- and medium-sized arteries and were only scarcely visible in large vessels $(\boldsymbol{D}-\boldsymbol{F})$. The histogram depicts the cumulative frequency of the thioflavin $\mathrm{S} /$ fibrinogen immunoreactivity according to different categories of vessel diameter $(\boldsymbol{H})$. Scale bars: $\boldsymbol{A}-\boldsymbol{C}, 10 \mu \mathrm{m}$; $D-F, 30 \mu \mathrm{m}$.

and $\operatorname{arcA} \beta$ mice (Fig. $6 B, C$, white arrows). Such intracellular lipofuscin aggregates were not observed in 4-month-old wt and transgenic mice and are known to be a sign of the aging of neurons (Dowson and Harris, 1981).

Fibrinogen accumulation was observed in vessels of 24month-old arcA $\beta$ mice (Fig. $6 \mathrm{C}$ ). The fibrinogen accumulation was always colocalized by thioflavin S-positive signal (Fig. 6G). thioflavin S/fibrinogen-immunoreactivity clearly differed between vessel categories. In small- and medium-sized arteries, thioflavin S/fibrinogen-positive signal was highly abundant, whereas in larger vessels it was scarcely visible (Fig. $6 D-F$ ). Some of the small vessels were filled by the fibrinogen deposition, indicating vessel stenosis (Fig. 6D). Quantitative assessment of immunoreactivity showed highest thioflavin S/fibrinogen-positive signal in vessels with a diameter between 20 and $40 \mu \mathrm{m}$ (Fig. $6 H$ ).

\section{Discussion}

Apart from the formation of parenchymal plaques, cerebral amyloidosis also involves deposition of $\mathrm{A} \beta$ peptides at vessels, which may lead to vascular remodeling as well as compromised function of the cerebrovasculature. In the $\operatorname{arc} A \beta$ transgenic mouse model, age-dependent aberrations in the structure and function of the cerebrovasculature have been reported (Princz-Kranz et al., 2010; Klohs et al., 2011; Merlini et al., 2011). We used MRA techniques to noninvasively assess cerebral vasculature. While TOF-MRA did not display any pathological changes, CE- $\mu$ MRA revealed a reduction in the density of the microvasculature in the $\operatorname{arcA} \beta$ mouse during advanced disease state.

TOF-MRA and CE- $\mu$ MRA can be used to assess different levels of the vascular tree. In TOF-MRA the signal depends on the blood flow (Lin et al., 1997), confining the method to the visualization of large cerebral arteries with high blood-flow rates. Highresolution TOF-MRA has been applied to transgenic mouse models of $\mathrm{AD}$ and $\mathrm{CAA}$, reporting prominent flow voids in the major arteries of aged APP overexpressing transgenic animals (Beckmann et al., 2003; Krucker et al., 2004; Thal et al., 2009; El Tannir El Tayara et al., 2010). In contrast, we did not detect flow voids in $\operatorname{arc} \mathrm{A} \beta$ mice. This is not surprising, because immunohistochemical analysis revealed that $\mathrm{A} \beta$ and fibrinogen deposition prevails in small- and medium-sized cerebral vessels, but effectively spares larger cerebral vessels. There was a trend toward a 
reduced distality count in TOF MRI of 24-month-old compared with 4-month-old mice, indicating a reduced blood flow in the older animals. El Tannir El Tayara et al. (2010) observed a reduction in the length of middle cerebral arteries both in aged APP/PS1 and wt control mice. This effect can be attributed to a decline in blood flow velocity due to aging (Vriens et al., 1989; Portman et al., 1992).

An intriguing finding in earlier TOF-MRA studies (Beckmann et al., 2003; Krucker et al., 2004; Thal et al., 2009; El Tannir El Tayara et al., 2010) was the observation of flow voids in extracranial vessels, such as the pterygopalatine artery, in APP overexpressing mice. As the transgene is expressed under the control of the Thy-1 promoter, $\mathrm{A} \beta$ deposition should be confined to intracerebral vessels. In the current study we observed similar flow voids, however, both in $\operatorname{arcA} \beta$ and wt mice of both age groups. This finding is consistent with earlier reports in APP/PS1 and age-matched wt mice (El Tannir El Tayara et al., 2010) and indicates that the flow voids observed are neither linked to the $\mathrm{A} \beta$ pathology nor the genetic trait. Instead, their occurrence might be attributed to susceptibility effects caused by the proximity of the tympanic bulba to the petrygopalatine artery (El Tannir El Tayara et al., 2010). Hence, in TOF-MRA the detectability of a vessel may be compromised either by reduced blood flow rates (Lin et al., 1997) or by local flow disturbances (Underwood and Mohiaddin, 1993). The technique appears of limited value for studying vascular remodeling in mouse models of CAA that affect medium-sized to small vessels characterized by low flow rates and potential abnormalities in flow pattern.

Therefore, we exploited the use of CE- $\mu$ MRA, which is independent of blood flow rates (Haacke et al., 1994; Lin et al., 1997; Bolan et al., 2006). As contrast agent we intravenously injected Endorem, which has a biphasic clearance kinetic (Majumdar et al., 1990). Approximately $56 \%$ of the injected dose was estimated to remain in the blood pool until the end of the postcontrast acquisition. Hence, contrast-agent filled blood vessels (both arteries and veins) could be clearly distinguished from stationary tissue.

By using CE- $\mu \mathrm{MRA}$, blood vessels smaller than the nominal image resolution can be detected, because the area affected by extravascular dephasing caused by the susceptibility difference between vessel and stationary tissue exceeds the vessel dimensions (Ogawa and Lee, 1990). Thus, significant changes in signal can be observed even when the vascular compartment comprises only a fraction of the voxel volume. We have used an algorithm that counted the vessels in the differences images when they fulfilled the criteria of Eq. 1. With this method, we found a significantly reduced number of intracortical vessels (radii of 20-80 $\mu \mathrm{m}$ ) in a mouse model of cerebral amyloidosis, indicating that noninvasive $\mathrm{CE}-\mu \mathrm{MRA}$ is sensitive in detecting vascular pathology affecting the microvasculature. Yet, the resolution of the method is not sufficient in resolving the status of capillaries with a diameter of $\sim 7-10 \mu \mathrm{m}$. Information about capillary density may be inferred using stochastic approaches such as vessel size imaging (Troprès et al., 2001).

Semiautomated analysis of CE- $\mu$ MRA datasets revealed a significant reduction in the number of intracortical vessels in 24month-old $\operatorname{arcA} \beta$ compared with aged-matched wt control mice, whereas there was no difference between transgenic and wt mice of 4 month of age. For verification of these findings, we investigated the density of intracortical vessels in corresponding brain section stained against the endothelial marker vWF. We observed no differences in expression levels of vWF among all groups, indicating that intracortical vessels in the 24-month-old $\operatorname{arcA} \beta$ mice have not been lost. However, as the vessels of these mice show some additional vWF immunoreactivity in nonendothelial structures, we cannot rule out that this increase in vWF expression level has masked a potential decrease in immunoreactivity due to vessel loss.

Vascular accumulation of $\mathrm{A} \beta$ has been associated with the deposition of fibrin which leads to the formation of clots that are structurally abnormal and, hence, resistant to fibrinolysis (Paul et al., 2007; Cortes-Canteli et al., 2010). Therefore, we used antifibrinogen immunohistochemistry to investigate whether the vessels in the 24-month-old $\operatorname{arcA} \beta$ mice were functionally altered. Aggregation of fibrin(ogen) was evident in cortical vessels of 24month-old $\operatorname{arcA} \beta$ mice, but not in 4 -month-old $\operatorname{arcA} \beta$ mice and in none of the age-matched wt controls. Some of the smaller blood vessels showed signs of stenosis due to the fibrin(ogen) accumulation. Furthermore, in the aged $\operatorname{arcA} \beta$ mice vWF immunoreactivity was observed in thrombus-like structures in crosssectioned blood vessels. As vWF displays also thrombogenic functions (Ruggeri, 2007) this indicates that the vessels of aged $\operatorname{arA} \beta$ are prone to thrombus formation.

The aggregation of fibrin(ogen) was always coincident with the vascular deposition of $\mathrm{A} \beta$, as indicated by the strong colocalization of thioflavin $S$ staining and fibrinogen immunoreactivity. Vessels without $A \beta$ accumulation, e.g., in the thalamus, were also devoid of fibrinogen. Furthermore, $A \beta /$ fibrinogen deposition seems to preferentially occur in vessels of a radius of $10-40 \mu \mathrm{m}$. Despite the fact that the absolute vessel diameter estimates of vessel diameter differ by factor 2-4 between the two methods (MRI and histology) due to tissue shrinkage during the histological preparation (Ratering et al., 2011), these vessels correspond to the same vessel category, which were found to be significantly reduced in number in the CE- $\mu$ MRA data. Together, these data indicate that the reduction of the microvascular density in $\operatorname{arcA} \beta$ mice is due to a lack of perfusion of existing blood vessels as a result of an altered thrombosis and fibrinolysis following vascular $\mathrm{A} \beta$ deposition.

In summary, high resolution $\mathrm{CE}-\mu \mathrm{MRA}$ revealed an agedependent reduction in the density of functional intracortical vessels (radii of $20-80 \mu \mathrm{m}$ ) in the $\operatorname{arcA} \beta$ mouse. This class of vessels is known to be preferentially affected by $\mathrm{A} \beta$ accumulation and seems to be prone to the formation of fibrin clots leading ultimately to vessel stenosis. These results suggest that the mechanism of fibrin accumulation might present therapeutic targets in slowing the progression of $\mathrm{A} \beta$ pathology. Due to its noninvasive nature, $\mathrm{CE}-\mu \mathrm{MRA}$ provides a powerful tool to assess the dynamics of cerebrovascular remodeling in the course of the disease process and to evaluate therapeutic interventions in mouse models of AD and CAA.

\section{References}

Baltes C, Radzwill N, Bosshard S, Marek D, Rudin M (2009) Micro MRI of the mouse brain using a novel $400 \mathrm{MHz}$ cryogenic quadrature RF probe. NMR Biomed 22:834-842.

Beckmann N, Schuler A, Mueggler T, Meyer EP, Wiederhold KH, Staufenbiel M, Krucker T (2003) Age-dependent cerebrovascular abnormalities and blood flow disturbances in APP23 mice modeling Alzheimer's disease. J Neurosci 23:8453-8459.

Benveniste H, Einstein G, Kim KR, Hulette C, Johnson GA (1999) Detection of neuritic plaques in Alzheimer's disease by magnetic resonance microscopy. Proc Natl Acad Sci U S A 96:14079-14084.

Bolan PJ, Yacoub E, Garwood M, Ugurbil K, Harel N (2006) In vivo microMRI of intracortical neurovasculature. Neuroimage 32:62-69.

Bouras C, Kövari E, Herrmann FR, Rivara CB, Bailey TL, von Gunten A, Hof PR, Giannakopoulos P (2006) Stereologic analysis of microvascular morphology in the elderly: Alzheimer disease pathology and cognitive status. J Neuropathol Exp Neurol 65:235-244.

Boxerman JL, Hamberg LM, Rosen BR, Weisskoff RM (1995) MR contrast 
due to intravascular magnetic susceptibility perturbations. Magn Reson Med 34:555-566.

Buée L, Hof PR, Bouras C, Delacourte A, Perl DP, Morrison JH, Fillit HM (1994) Pathological alterations of the cerebral microvasculature in Alzheimer's disease and related dementing disorders. Acta Neuropathol 87: 469-480.

Cadavid D, Mena H, Koeller K, Frommelt RA (2000) Cerebral beta amyloid angiopathy is a risk factor for cerebral ischemic infarction. A case control study in human brain biopsies. J Neuropathol Exp Neurol 59:768-773.

Calhoun ME, Burgermeister P, Phinney AL, Stalder M, Tolnay M, Wiederhold KH, Abramowski D, Sturchler-Pierrat C, Sommer B, Staufenbiel M, Jucker M (1999) Neuronal overexpression of mutant amyloid precursor protein results in prominent deposition of cerebrovascular amyloid. Proc Natl Acad Sci U S A 96:14088-14093.

Cortes-Canteli M, Paul J, Norris EH, Bronstein R, Ahn HJ, Zamolodchikov D, Bhuvanendran S, Fenz KM, Strickland S (2010) Fibrinogen and betaamyloid association alters thrombosis and fibrinolysis: a possible contributing factor to Alzheimer's disease. Neuron 66:695-709.

de la Torre JC (1997) Hemodynamic consequences of deformed microvessels in the brain in Alzheimer's disease. Ann NY Acad Sci 826:75-91.

Dowson JH, Harris SJ (1981) Quantitative studies of the autofluorescence derived from neuronal lipofuscin. J Microsc 123:249-258.

El Tannir El Tayara N, Delatour B, Volk A, Dhenain M (2010) Detection of vascular alterations by in vivo magnetic resonance angiography and histology in APP/PS1 mouse model of Alzheimer's disease. MAGMA 23:53-64.

Fischer VW, Siddiqi A, Yusufaly Y (1990) Altered angioarchitecture in selected areas of brains with Alzheimer's disease. Acta Neuropathol 79:672-679.

Franceschi M, Alberoni M, Bressi S, Canal N, Comi G, Fazio F, Grassi F, Perani D, Volonté MA (1995) Correlations between cognitive impairment, middle cerebral artery flow velocity and cortical glucose metabolism in the early phase of Alzheimer's disease. Dementia 6:32-38.

Gilbert JJ, Vinters HV (1983) Cerebral amyloid angiopathy: incidence and complications in the aging brain. I. Cerebral hemorrhage. Stroke 14:915-923.

Glenner GG, Henry JH, Fujihara S (1981) Congophilic angiopathy in the pathogenesis of Alzheimer's degeneration. Ann Pathol 1:120-129.

Haacke EM, Hopkins A, Lai S, Buckley P, Friedman L, Meltzer H, Hedera P, Friedland R, Klein S, Thompson L, Detterman D, Tkach J, Lewin JS (1994) 2D and 3D high resolution gradient echo functional imaging of the brain: venous contributions to signal in motor cortex studies. NMR Biomed 7:54-62.

Haglund M, Passant U, Sjöbeck M, Ghebremedhin E, Englund E (2006) Cerebral amyloid angiopathy and cortical microinfarcts as putative substrates of vascular dementia. Int J Geriatr Psychiatry 21:681-687.

Herzig MC, Winkler DT, Burgermeister P, Pfeifer M, Kohler E, Schmidt SD, Danner S, Abramowski D, Stürchler-Pierrat C, Bürki K, van Duinen SG, Maat-Schieman ML, Staufenbiel M, Mathews PM, Jucker M (2004) Abeta is targeted to the vasculature in a mouse model of hereditary cerebral hemorrhage with amyloidosis. Nat Neurosci 7:954-960.

Klohs J, Deistung A, Schweser F, Grandjean J, Dominietto M, Waschkies C, Nitsch RM, Knuesel I, Reichenbach J, Rudin M (2011) Detection of cerebral microbleeds with quantitative susceptibility mapping in the ArcAbeta mouse model of cerebral amyloidosis. J Cereb Blood Flow Metab 31:2282-2292.

Knobloch M, Konietzko U, Krebs DC, Nitsch RM (2007) Intracellular Abeta and cognitive deficits precede beta-amyloid deposition in transgenic arcAbeta mice. Neurobiol Aging 28:1297-1306.

Kouznetsova E, Klingner M, Sorger D, Sabri O, Grossmann U, Steinbach J, Scheunemann M, Schliebs R (2006) Developmental and amyloid plaque-related changes in cerebral cortical capillaries in transgenic Tg2576 Alzheimer mice. Int J Dev Neurosci 24:187-193.

Krucker T, Schuler A, Meyer EP, Staufenbiel M, Beckmann N (2004) Magnetic resonance angiography and vascular corrosion casting as tools in biomedical research: application to transgenic mice modeling Alzheimer's disease. Neurol Res 26:507-516.

Lee GD, Aruna JH, Barrett PM, Lei DL, Ingram DK, Mouton PR (2005) Stereological analysis of microvascular parameters in a double transgenic model of Alzheimer's disease. Brain Res Bull 65:317-322.

Lin W, Abendschein DR, Celik A, Dolan RP Lauffer RB, Walovitch RC, Haacke EM (1997) Intravascular contrast agent improves magnetic res- onance angiography of carotid arteries in minipigs. J Magn Reson Imaging 7:963-971.

Majumdar S, Zoghbi SS, Gore JC (1990) Pharmacokinetics of superparamagnetic iron-oxide MR contrast agents in the rat. Invest Radiol 25:771-777.

Marchesi VT (2011) Alzheimer's dementia begins as a disease of small blood vessels, damaged by oxidative-induced inflammation and dysregulated amyloid metabolism: implications for early detection and therapy. FASEB J 25:5-13.

Merlini M, Meyer EP, Ulmann-Schuler A, Nitsch RM (2011) Vascular $\beta$-amyloid and early astrocyte alterations impair cerebrovascular function and cerebral metabolism in transgenic $\operatorname{arcA} \beta$ mice. Acta Neuropathol 122:293-311.

Miao J, Xu F, Davis J, Otte-Höller I, Verbeek MM, Van Nostrand WE (2005) Cerebral microvascular amyloid beta protein deposition induces vascular degeneration and neuroinflammation in transgenic mice expressing human vasculotropic mutant amyloid beta precursor protein. Am J Pathol 167:505-515.

Moody DM, Brown WR, Challa VR, Ghazi-Birry HS, Reboussin DM (1997) Cerebral microvascular alterations in aging, leukoaraiosis, and Alzheimer's disease. Ann NY Acad Sci 26:103-116.

Ogawa S, Lee TM (1990) Magnetic resonance imaging of blood vessels at high fields: in vivo and in vitro measurements and image simulation. Magn Reson Med 16:9-18.

Ogawa S, Lee TM, Nayak AS, Glynn P (1990) Oxygenation-sensitive contrast in magnetic resonance image of rodent brain at high magnetic fields. Magn Reson Med 14:68-78.

Paul J, Strickland S, Melchor JP (2007) Fibrin deposition accelerates neurovascular damage and neuroinflammation in mouse models of Alzheimer's disease. J Exp Med 204:1999-2008.

Portman MA, Cooper TG, Potchen EJ (1992) Physiologic alterations in cranial blood flow demonstrated by magnetic resonance angiography. Invest Radiol 27:240-244.

Princz-Kranz FL, Mueggler T, Knobloch M, Nitsch RM, Rudin M (2010) Vascular response to acetazolamide decreases as a function of age in the arcA beta mouse model of cerebral amyloidosis. Neurobiol Dis 40:284-292.

Ratering D, Baltes C, Lohmann C, Matter CM, Rudin M (2011) Accurate assessment of carotid artery stenosis in atherosclerotic mice using accelerated high-resolution 3D magnetic resonance angiography. MAGMA 24:9-18.

Reese T, Bochelen D, Sauter A, Beckmann N, Rudin M (1999) Magnetic resonance angiography of the rat cerebrovascular system without the use of contrast agents. NMR Biomed 12:189-196.

Reichenbach JR, Venkatesan R, Schillinger DJ, Kido DK, Haacke EM (1997) Small vessels in the human brain: MR venography with deoxyhemoglobin as an intrinsic contrast agent. Radiology 204:272-277.

Ruggeri ZM (2007) The role of von Willebrand factor in thrombus formation. Thromb Res 120 [Suppl 1]:S5-S9.

Ryu JK, McLarnon JG (2009) A leaky blood-brain barrier, fibrinogen infiltration and microglial reactivity in inflamed Alzheimer's disease brain. J Cell Mol Med 13:2911-2925.

Thal DR, Capetillo-Zarate E, Larionov S, Staufenbiel M, Zurbruegg S, Beckmann N (2009) Capillary cerebral amyloid angiopathy is associated with vessel occlusion and cerebral blood flow disturbances. Neurobiol Aging 30:1936-1948.

Troprès I, Grimault S, Vaeth A, Grillon E, Julien C, Payen JF, Lamalle L, Décorps M (2001) Vessel size imaging. Magn Reson Med 45:397-408.

Underwood RS, Mohiaddin RH (1993) Magnetic resonance imaging of atherosclerotic vascular disease. Am J Hypertens 6:335S-339S.

Vriens EM, Kraaier V, Musbach M, Wieneke GH, van Huffelen AC (1989) Transcranial pulsed Doppler measurements of blood velocity in the middle cerebral artery: reference values at rest and during hyperventilation in healthy volunteers in relation to age and sex. Ultrasound Med Biol 15:1-8.

Weller RO, Subash M, Preston SD, Mazanti I, Carare RO (2008) Perivascular drainage of amyloid-beta peptides from the brain and its failure in cerebral amyloid angiopathy and Alzheimer's disease. Brain Pathol 18:253-266.

Weller RO, Boche D, Nicoll JA (2009) Microvascular changes and cerebral amyloid angiopathy in Alzheimer's disease and their potential impact on therapy. Acta Neuropathol 118:87-102. 